PROCEEDINGS OF THE

AMERICAN MATHEMATICAL SOCIETY

Volume 132, Number 1, Pages 251-259

S 0002-9939(03)07026-6

Article electronically published on May 28, 2003

\title{
SMALL SUBSETS OF THE REALS AND TREE FORCING NOTIONS
}

\author{
MARCIN KYSIAK AND TOMASZ WEISS \\ (Communicated by Carl G. Jockusch, Jr.)
}

\begin{abstract}
We discuss the question of which properties of smallness in the sense of measure and category (e.g. being a universally null, perfectly meager or strongly null set) imply the properties of smallness related to some tree forcing notions (e.g. the properties of being Laver-null or Miller-null).
\end{abstract}

\section{Notation And terminology}

Most of the notation used in this paper is standard.

The symbol $\omega$ denotes the set of natural numbers. By $A^{\omega}$ we denote the set of all infinite sequences of elements of $A$ (where $A$ will always be equal to $\omega$ or $2=\{0,1\}$ ). The symbol $A^{<\omega}$ stands for the set of all finite sequences of elements of $A$. For $s \in A^{<\omega},[s]$ is the set of all infinite sequences of elements of $A$ which extend $s$ :

$$
[s]=\left\{x \in A^{\omega}: s \subseteq x\right\} .
$$

We say that a set $T \subseteq A^{<\omega}$ is a tree if $T$ is closed under restrictions, i.e.,

$$
\forall s \in T \forall n \leq|s| s\lceil n \in T .
$$

Every tree is naturally ordered by the reverse inclusion (so $s \leq t$ if, and only if, $s$ extends $t$, for $s, t$ being elements of a tree). If $T \subseteq A^{<\omega}$ is a tree, then by $[T]$ we denote its body, i.e. the set of all infinite branches of $T$ :

$$
[T]=\left\{x \in A^{\omega}: \forall n \in \omega x\lceil n \in T\} .\right.
$$

For a tree $T$, we define $\operatorname{Split}(T)$ to be the set

$$
\left\{s \in T:\left|\left\{n \in \omega: s^{\frown} n \in T\right\}\right|>1\right\} .
$$

By $\operatorname{Split}^{n}(T)$ we will denote the $n$-th level of $\operatorname{Split}(T)$ :

$$
\operatorname{Split}^{n}(T)=\{s \in \operatorname{Split}(T): \mid\{k<|s|: s\lceil k \in \operatorname{Split}(T)\} \mid=n\} .
$$

Received by the editors June 11, 2002 and, in revised form, September 3, 2002.

2000 Mathematics Subject Classification. Primary 03E35, 28E15.

Key words and phrases. Perfectly meager set, universally null set, strong measure zero set, Laver forcing, Miller forcing.

The first author is a Ph.D. student at the Institute of Mathematics of the Polish Academy of Sciences. A part of this work is likely to be included in his doctorate written under the supervision of P. Zakrzewski.

(C)2003 American Mathematical Society 
We say that a tree $T$ is well-founded, if $[T]=\emptyset$, i.e. if every chain in $T$ is finite. For a well-founded tree $T$, we define

$$
\widetilde{T}=\left\{x \in A^{\omega}: \exists n \in \omega x\lceil n \text { is a minimal element in } T\} .\right.
$$

We will say that a tree is well-pruned if every maximal chain in $T$ is infinite. Unless otherwise stated (which will be the case for well-founded trees), we will assume that every tree in question is well-pruned.

The spaces $\omega^{\omega}$ and $2^{\omega}$ are given the standard product topologies. In the case of $2^{\omega}$ we also introduce a group structure in this space, treating it as the countable product of the group $\mathbb{Z}_{2}$. We will also consider the standard product probability measure on $2^{\omega}$, which will be referred to as the Lebesgue measure.

For $f, g \in \omega^{\omega}$, we write $f \leq^{*} g$ if $f(n) \leq g(n)$, for all but finitely many $n \in \omega$. The cardinal number $\mathfrak{b}$ is the smallest size of an unbounded family, i.e. a family $\mathcal{F} \subseteq \omega^{\omega}$ such that for every $g \in \omega^{\omega}$, there exists $f \in \mathcal{F}$ such that $f \mathbb{Z}^{*} g$.

The cardinal number continuum, i.e. the cardinality of the set of real numbers, is denoted by $\mathfrak{c}$.

\section{INTRODUCTION AND BASIC DEFINITIONS}

Let us begin with a few definitions of small sets in the sense of measure and category.

Definition 2.1. A subset $X$ of a metric space is called strongly null (or strong measure zero set, $X \in \mathcal{S N}$ ) if for every sequence of positive reals $\left\langle\varepsilon_{n}: n \in \omega\right\rangle$, there exists a sequence of open sets $\left\langle I_{n}: n \in \omega\right\rangle$ such that $\operatorname{diam}\left(I_{n}\right) \leq \varepsilon_{n}$ and $X \subseteq \bigcup_{n} I_{n}$

Definition 2.2. A subset $X$ of a Polish space $Y$ is called universally null $(X \in \mathcal{U N})$ if it is null with respect to any Borel probability measure on $Y$ vanishing on points.

Definition 2.3. A set $X \subseteq 2^{\omega}$ is called strongly meager $(X \in \mathcal{S M})$ if for every full Lebesgue measure set $G$, there exists $t \in 2^{\omega}$ such that $X \subseteq t+G$.

Definition 2.4. A set $X \subseteq 2^{\omega}$ is called very meager $(X \in \mathcal{V} \mathcal{M})$ if for every full Lebesgue measure set $G$, there exists $T \in\left[2^{\omega}\right]^{\omega}$ such that $X \subseteq T+G$.

Definition 2.5. A subset $X$ of a Polish space is called perfectly meager $(X \in \mathcal{P} \mathcal{M})$ if for every perfect set $P$, the set $X \cap P$ is meager in $P$.

It is known (and in some cases obvious) that the following inclusions hold between these classes in the space $2^{\omega}$ :

- $\mathcal{S N} \subsetneq \mathcal{U N} \subsetneq \mathcal{N}$

- $\mathcal{S M} \subseteq \mathcal{V} \mathcal{M} \subsetneq \mathcal{P} \mathcal{M} \subsetneq \mathcal{M}$.

Moreover, both strongly meager and very meager sets can be considered to be a "category analogue" of strongly null sets (see [1] and [4]). Similarly, perfectly meager sets are a category analogue of universally null sets.

Definition 2.6. A tree $T \subseteq \omega^{<\omega}$ is called

- a perfect tree if

$$
\forall s \in T \exists t \in T \quad t \supseteq s \wedge\left|\left\{n \in \omega: t^{\frown} n \in T\right\}\right|>1,
$$

- a Laver tree if

$$
\exists s \in T \forall t \in T \quad t \subseteq s \vee|\{n \in \omega: t \frown n \in T\}|=\omega,
$$


- a Miller tree (or a superperfect tree) if

$$
\forall s \in T \exists t \in T \quad t \supseteq s \wedge\left|\left\{n \in \omega: t^{\frown} n \in T\right\}\right|=\omega .
$$

In other words, $T$ is a perfect tree if it splits somewhere below every node, $T$ is a Miller tree if somewhere below every node it splits into infinitely many successors, and $T$ is a Laver tree if it has a stem $s$, and below this stem it splits into infinitely many successors at every node.

Let $\mathbb{L}, \mathbb{M}, \mathbb{S}$ denote the classes of Laver, Miller and perfect trees, respectively. Then obviously $\mathbb{L} \subseteq \mathbb{M} \subseteq \mathbb{S}$.

Definition 2.7. Let $X \subseteq \omega^{\omega}$. We say that

- $X$ has the $l_{0}$-property if

$$
\forall T \in \mathbb{L} \exists T^{\prime} \in \mathbb{L}\left(T^{\prime} \subseteq T \wedge\left[T^{\prime}\right] \cap X=\emptyset\right),
$$

- $X$ has the $m_{0}$-property if

$$
\forall T \in \mathbb{M} \exists T^{\prime} \in \mathbb{M}\left(T^{\prime} \subseteq T \wedge\left[T^{\prime}\right] \cap X=\emptyset\right),
$$

- $X$ has the $s_{0}$-property if

$$
\forall T \in \mathbb{S} \exists T^{\prime} \in \mathbb{S}\left(T^{\prime} \subseteq T \wedge\left[T^{\prime}\right] \cap X=\emptyset\right) .
$$

It can be proved in ZFC (see [2]) that there is no implication between any two of the properties $l_{0}, m_{0}, s_{0}$.

For the purpose of our paper we would also like to extend the notions of the $l_{0^{-}}$, $m_{0^{-}}$and $s_{0}$-properties to subsets of $2^{\omega}$. This is motivated by the fact that while the space $\omega^{\omega}$ has a nice combinatorial structure required to consider these classes of sets, the classes of sets like $\mathcal{S M}$ and $\mathcal{V M}$ are usually studied in the space $2^{\omega}$.

Let $\omega^{\uparrow \omega}$ denote the set of all strictly increasing elements of $\omega^{\omega}$. One can easily check that the function $\phi_{1}: \omega^{\omega} \rightarrow \omega^{\uparrow \omega}$, defined as

$$
\phi_{1}:\left\langle x_{0}, x_{1}, x_{2}, \ldots\right\rangle \longmapsto\left\langle x_{0}+0, x_{0}+x_{1}+1, x_{0}+x_{1}+x_{2}+2, \ldots\right\rangle,
$$

is a homeomorphism. Moreover, the function $\phi_{2}: \omega^{\uparrow \omega} \rightarrow 2^{\omega}$ which identifies an increasing function with the characteristic function of its range is a homeomorphism between $\omega^{\uparrow \omega}$ and $[\omega]^{\omega} \subseteq 2^{\omega}$. Thus $\phi=\phi_{2} \circ \phi_{1}$ is a natural embedding of $\omega^{\omega}$ onto a co-countable subset of $2^{\omega}$.

Definition 2.8. A set $X \subseteq 2^{\omega}$ has the $s_{0}$-property ( $l_{0}$-property, $m_{0}$-property) if $\phi^{-1}[X]$ has the $s_{0}$-property ( $l_{0}$-property, $m_{0}$-property, resp.) in $\omega^{\omega}$.

Remark 2.9. A better known way of defining the $s_{0}$-property in $2^{\omega}$ is simply to reformulate our definition of the $s_{0}$-property in $\omega^{\omega}$ in terms of perfect trees on $2^{<\omega}$ : a set $X \subseteq 2^{\omega}$ has the $s_{0}$-property if for every perfect tree $T \subseteq 2^{<\omega}$, there exists a perfect tree $T^{\prime} \subseteq T$ such that $X \cap\left[T^{\prime}\right]=\emptyset$. We would like to point out that these two definitions are equivalent. This easily follows from the Perfect Set Theorem for Borel sets and from the fact that the bodies of the perfect trees (either on $\omega^{<\omega}$ or $2^{<\omega}$ ) are precisely perfect sets (in $\omega^{\omega}$ or $2^{\omega}$, respectively).

Remark 2.10. We had to fix our homeomorphism $\phi$ to have a logically correct definition. Our choice seems to follow the idea of Brendle's definition from [2].

One can check that at least the definitions of the $m_{0^{-}}$and $s_{0}$-properties in $2^{\omega}$ do not depend on the choice of $\phi$, i.e. we would obtain equivalent definitions if we considered another homeomorphism instead. This is true because the concepts 
of a perfect and a Miller tree can be expressed in purely topological language (see Remark 2.9] and Lemma 3.4, for instance).

It is not clear whether the definition of the $l_{0}$-property in $2^{\omega}$ depends on the choice of a particular homeomorphism. Nevertheless, all the results from our paper remain true if we consider another homeomorphism instead.

Let us also observe that, since $\phi$ is a homeomorphism between $\omega^{\omega}$ and a cocountable subset of $2^{\omega}$, a set $X \subseteq \omega^{\omega}$ is universally null (perfectly meager) in $\omega^{\omega}$ if, and only if, $\phi[X]$ is universally null (perfectly meager, resp.) in $2^{\omega}$. Thus, if we write about universally null (or perfectly meager) sets without specifying the space we are working in, then we mean that the result is general, i.e. it holds for universally null (or perfectly meager) subsets of $\omega^{\omega}$ and $2^{\omega}$.

A classical result (see [6]) says that:

\section{Theorem 2.11.}

- Every perfectly meager set has the $s_{0}$-property.

- Every universally null set has the $s_{0}$-property.

It follows, in particular, that all the properties mentioned in Definitions 2.1 2.5 imply the $s_{0}$-property.

In our paper we continue the study from [9], discussing the possibility of proving "l$l_{0}$ and $m_{0}$ versions" of this theorem for perfectly meager, universally null and strongly null sets.

\section{ON THE CATEGORY SIDE}

The following theorem was already proved in 9$]$ :

Theorem 3.1. Every very meager set $X \subseteq 2^{\omega}$ has both the $l_{0}$ - and the $m_{0}$-property.

We begin with an observation already announced in [9].

Theorem 3.2. Every perfectly meager set has the $m_{0}$-property.

We need the following lemma:

Lemma 3.3. For every meager set $F \subseteq \omega^{\omega}$, there exists a Miller tree $T$ such that $[T] \cap F=\emptyset$.

Proof. Without loss of generality we can assume that $F=\bigcup_{n \in \omega} F_{n}$, where each $F_{n}$ is closed nowhere dense.

We will inductively construct an increasing sequence $\left\langle T_{n}: n \in \omega\right\rangle$ of well-founded trees such that:

- $\widetilde{T_{n+1}} \subseteq \widetilde{T_{n}}$

- $\widetilde{T}_{n} \cap F_{n}=\emptyset$,

- $T=\bigcup_{n \in \omega} T_{n}$ is a Miller tree.

Clearly, the first two conditions imply that $[T] \cap F=\emptyset$.

Let $s \in \omega^{<\omega}$ be any sequence such that $[s] \cap F_{0}=\emptyset$. Obviously, $[s]=\bigcup_{n}[s \frown n]$, so let $T_{0}$ be the smallest well-founded tree containing $\{s \frown n: n \in \omega\}$.

Suppose that we have constructed $T_{n}$. For every minimal element $s$ in $T_{n}$, we can find $r_{s} \supseteq s$ such that $\left[r_{s}\right] \cap F_{n+1}=\emptyset$. As before, $\left[r_{s}\right]=\bigcup_{n}\left[r_{s}^{\frown n}\right]$. Now let $T_{n+1}$ be the smallest well-founded tree containing all $r_{s}^{\frown} n$, for all minimal elements in $T_{n}$. It is easy to check that $T_{n+1}$ is as needed. 
We will say that a closed set $F \subseteq \omega^{\omega}$ is nowhere compact if for every clopen set $U \subseteq \omega^{\omega}$, the set $U \cap F$ is not compact whenever $U \cap F \neq \emptyset$. We leave the following easy lemma as an exercise to the reader:

Lemma 3.4. A closed set $F \subseteq \omega^{\omega}$ is nowhere compact if and only if there exists a Miller tree $T$ such that $F=[T]$.

Proof of Theorem 3.2. Let $X \subseteq \omega^{\omega}$ be a perfectly meager set and let $T$ be any Miller tree. As $[T]$ is a perfect set, $[T] \cap X$ is meager in $[T]$. But $[T]$ is homeomorphic to $\omega^{\omega}$, so applying Lemma 3.3 in $[T]$ and using Lemma 3.4, we see that there exists a nowhere compact set $H \subseteq[T]$ disjoint with $X$. But if $H$ is a nowhere compact set in $[T]$, then it is also a nowhere compact set in $\omega^{\omega}$, so by Lemma 3.4 again, $H=\left[T^{\prime}\right] \subseteq[T]$, for some Miller tree $T^{\prime} \subseteq T$.

It turns out that the analogous assertion for the $l_{0}$-property need not be true. Recall that an $\omega_{1}$-scale is an increasing (in the sense of $\leq^{*}$ ) and dominating sequence of elements of $\omega^{\omega}$ of length $\omega_{1}$.

Lemma 3.5. Assume $\mathrm{CH}$. Then there exists an $\omega_{1}$-scale which intersects $[T]$, for every Laver tree $T$.

Proof. Assume CH. Let $\left\langle T_{\alpha}: \alpha<\omega_{1}\right\rangle$ be an enumeration of all Laver trees and let $\left\langle g_{\alpha}: \alpha<\omega_{1}\right\rangle$ be an enumeration of $\omega^{\omega}$. Observe that if $T$ is a Laver tree, then $[T]$ is a dominating family. Thus, proceeding by induction, we may always pick $f_{\alpha} \in\left[T_{\alpha}\right]$ such that

$$
\left(\left\{f_{\xi}: \xi<\alpha\right\} \cup\left\{g_{\alpha}\right\}\right)<{ }^{*} f_{\alpha} .
$$

Obviously, $X=\left\{f_{\alpha}: \alpha<\omega_{1}\right\}$ is an $\omega_{1}$-scale. Moreover, by the choice of $f_{\alpha}, X$ intersects $[T]$, for every Laver tree $T$.

One can easily check that the proof of the previous lemma also works under the assumption $\mathfrak{b}=\mathfrak{c}$.

Theorem 3.6. Assume $\mathrm{CH}$. Then not every perfectly meager set has the $l_{0}$ property.

Proof. It was proved in 8 that every $\omega_{1}$-scale has a certain very strong combinatorial property which implies its perfect meagerness. Thus, if we consider an $\omega_{1}$-scale as in Lemma 3.5 then it does not have the $l_{0}$-property.

It turns out that such an example cannot be constructed in ZFC only.

Proposition 3.7. It is relatively consistent with ZFC that every perfectly meager set has the $l_{0}$-property.

Proof. It is known (see [5]) that in the iterated Sacks model, every perfectly meager set has size smaller than continuum. On the other hand, one can show in ZFC that every set of cardinality smaller than continuum has the $l_{0}$-property. It follows from the fact that for every Laver tree $T$, the set $[T]$ can be partitioned into continuum many disjoint sets of the form $\left[S_{\xi}\right]$, for some family $\left\{S_{\xi}: \xi<\mathfrak{c}\right\} \subseteq \mathbb{L}$. 


\section{ON THE MEASURE SIDE}

In this section we will discuss relations between the classes of strongly and universally null sets and the classes of sets with the $l_{0}$ - and the $m_{0}$-property.

Let us introduce some additional terminology. Let $T \subseteq \omega^{<\omega}$ be any tree. We will say that a sequence $\left\{t_{n}: n \in \omega\right\} \subseteq \omega^{<\omega}$ kills $s \in T$ if there exists $n \in \omega$ such that $t_{n} \subseteq s$.

Fix such a sequence $\left\{t_{n}: n \in \omega\right\}$ for a moment. Let $T^{\prime}$ be the set of those $t \in T$ which are not killed by this sequence. Observe that $T^{\prime}$ is a subtree of $T$. Moreover, $\left[T^{\prime}\right]=[T] \backslash \bigcup_{n}\left[t_{n}\right]$.

Let us begin with the following observation which is left to the reader:

Proposition 4.1. Suppose that the following fact holds for a tree $T$ : $\operatorname{stem}(T)$ is not killed by $\left\{t_{n}: n \in \omega\right\}$ and if $s \in \operatorname{Split}^{m}(T)$ is not killed by $\left\{t_{n}: n \in \omega\right\}$, then there are infinitely many $s^{\prime} \in$ Split $^{m+1}(T)$ extending $s$ which are not killed by $\left\{t_{n}: n \in \omega\right\}$ either.

Let $T^{\prime}$ be the tree consisting of those $s \in T$ which are not killed by $\left\{t_{n}: n \in \omega\right\}$. Then if $T$ is a Laver (Miller) tree, then $T^{\prime}$ is a Laver (Miller, resp.) tree as well.

Let $d$ be the standard metric on $\omega^{\omega}$ defined by the following formula:

$$
d(x, y)=\frac{1}{\min \{n \in \omega: x(n) \neq y(n)\}+1} .
$$

Theorem 4.2. Every strongly null subset of $\left(\omega^{\omega}, d\right)$ has the $l_{0}$-property.

Proof. Let $X$ be a strongly null subset of $\left(\omega^{\omega}, d\right)$ and let $T$ be any Laver tree. Using Proposition 4.1 we will construct a sequence $\left\langle\varepsilon_{n}: n \in \omega\right\rangle$ such that if $\left|t_{n}\right|=\frac{1}{\varepsilon_{n}}$, then the tree $T^{\prime}$ consisting of those $t \in T$ which are not killed by $\left\{t_{n}: n \in \omega\right\}$ will be a Laver tree.

Observe first that this really finishes the proof. Indeed, we can choose $\left\langle t_{n}: n \in \omega\right\rangle$ such that $X \subseteq \bigcup_{n}\left[t_{n}\right]$. Then $T^{\prime} \subseteq T$ is a Laver tree such that $\left[T^{\prime}\right] \cap X=\emptyset$.

Let $\varepsilon_{n}=\frac{1}{|\operatorname{stem}(T)|+n+1}$. It is easy to observe that if $\left|t_{n}\right|=\left(\varepsilon_{n}\right)^{-1}$, then the assumption of Proposition 4.1 holds, since if $s \in \operatorname{Split}^{m}(T)$ is not killed, then at most one $s^{\prime} \in \operatorname{Split}^{m+1}(T)$ extending $s$ is killed.

Theorem 4.3. Every strongly null subset of $\left(\omega^{\omega}, d\right)$ has the $m_{0}$-property.

Observe that, as in the proof of Theorem 4.2, it is sufficient to find a sequence $\left\langle\varepsilon_{n}: n \in \omega\right\rangle$ such that for a given Miller tree $T$, the assumption of Proposition 4.1 is satisfied, if only $\left|t_{n}\right|=\left(\varepsilon_{n}\right)^{-1}$. The construction of this sequence will be more complicated in this case. We will need the following lemma (we would like to thank P. Zakrzewski for a remark simplifying its proof):

Lemma 4.4. For every countable family $\mathcal{F} \subseteq \omega^{\omega}$, there exists $g \in \omega^{\omega}$ such that for any injection $i: \omega \rightarrow \omega$ and for every $f \in \mathcal{F}$, we have

$$
g \circ i \not \mathbb{Z}^{*} f \text {. }
$$

Proof. Let $g$ be a strictly increasing function such that

$$
\forall f \in \mathcal{F} \forall^{\infty} n \quad f(n)<g(n) .
$$

We will show that $g$ is as needed. 
Let $\left\langle i_{n}: n \in \omega\right\rangle$ be any injection and suppose that $\forall^{\infty} n g\left(i_{n}\right) \leq f(n)$. We claim that

$$
\exists^{\infty} n i_{n} \geq n
$$

Indeed, suppose that $\forall^{\infty} n i_{n}<n$. Let $N \in \omega$ be such that $\forall n \geq N i_{n}<n$ and put $K=\max \left(\left\{i_{k}: k<N\right\} \cup\{N\}\right)+1$. Notice that $\forall k<K i_{k}<K$, so $\left\{i_{k}: k<K\right\}=K$. But then $i_{K} \geq K$ which contradicts the fact that $K \geq N$.

Now, as $\exists^{\infty} n i_{n} \geq n$, we have

$$
\exists^{\infty} n f(n) \geq g\left(i_{n}\right) \geq g(n)
$$

which contradicts the choice of $g$.

Proof of Theorem 4.3. Let $T$ be a Miller tree. For every $s \in \operatorname{Split}(T)$ (say $s \in$ Split $\left.{ }^{m}(T)\right)$, let $f_{s}: \omega \rightarrow \omega$ be a function such that $f_{s}(n)=\left|s_{n}\right|$, where $\left\{s_{n}\right.$ : $n \in \omega\}$ is an injective enumeration of those $s^{\prime} \in \operatorname{Split}^{m+1}(T)$ which extend $s$. In other words, $f_{s}$ codes the lengths of all immediate successors of $s$ in $\operatorname{Split}(T)$. Let $f_{s}^{N}(k)=f_{s}(N+k)$. Apply Lemma 4.4 to the family

$$
\mathcal{F}=\left\{f_{s}^{N}: s \in \operatorname{Split}(T), N \in \omega\right\}
$$

to obtain $g \in \omega^{\omega}$ and let $\varepsilon_{n}=(g(n))^{-1}$. We can assume that $g(0)>|\operatorname{stem}(T)|$. Suppose that we have a sequence $\left\{t_{n}: n \in \omega\right\}$ such that $\left|t_{n}\right|=\left(\varepsilon_{n}\right)^{-1}=g(n)$. We claim that the assumption of Proposition 4.1 is satisfied.

As we have assumed that $g(0)>|\operatorname{stem}(T)|$, the stem of our tree is not killed. Take $s \in \operatorname{Split}^{m}(T)$ which is not killed by $\left\{t_{n}: n \in \omega\right\}$. Let $\left\{s_{n}: n \in \omega\right\}$ be an injective enumeration of all $s^{\prime} \in$ Split $^{m+1}$ which extend $s$. Suppose that almost all of $s_{n}$ were killed. Then for some $N \in \omega$ and for all of the $n \in \omega$, the sequence $s_{N+n}$ extends $t_{i_{n}}$, for some $i_{n} \in \omega$. Observe that, since $s$ was not killed, $i_{n} \neq i_{n^{\prime}}$, for $n \neq n^{\prime}$, so the function $i: n \mapsto i_{n}$ is an injection. But this contradicts the choice of $g$, since we have

$$
\forall n g\left(i_{n}\right)=\left|t_{i_{n}}\right| \leq\left|s_{N+n}\right|=f_{s}^{N}(n) .
$$

Now let $\left\{t_{n}: n \in \omega\right\}$ be such that $\left|t_{n}\right|=\left(\varepsilon_{n}\right)^{-1}$ and $X \subseteq \bigcup_{n}\left[t_{n}\right]$. Proposition 4.1 gives us a Miller tree $T^{\prime} \subseteq T$ such that $\left[T^{\prime}\right] \cap X=\emptyset$.

So far we have been working with the standard metric on $\omega^{\omega}$. It turns out that the choice of the metric in this space may be important in questions concerning strongly null subsets of $\omega^{\omega}$. As we will see, subsets of $\omega^{\omega}$ which are strongly null with respect to the metric inherited from the standard metric on the Cantor space need not have the $l_{0}$-property or the $m_{0}$-property. A similar phenomenon was observed by J. B. Brown in [3], where he considered the question of whether strongly null subsets of $\omega^{\omega}$ have the so-called $c r_{0}$-property.

Observe that strongly null subsets of $2^{\omega}$ can be seen (modulo a countable set) as strongly null subsets of $\omega^{\omega}$ with a suitable metric transformed via $\phi^{-1}$ from $\phi\left[\omega^{\omega}\right] \subseteq 2^{\omega}$.

Theorem 4.5. Assume CH. Then not every strongly null subset of $2^{\omega}$ has the $l_{0}$-property.

Proof. It is well known that an image by $\phi$ of every $\omega_{1}$-scale is strongly null in $2^{\omega}$. Lemma 3.5 finishes the proof.

As every strongly null set in $2^{\omega}$ is universally null, we get

Corollary 4.6. Under $\mathrm{CH}$ not every universally null set has the $l_{0}$-property. 
For subsets $X, Y$ of a metric space, we say that $X$ is concentrated on $Y$ if for every open set $U \supseteq Y$, we have $|X \backslash U| \leq \omega$. It is easy to check that if a set $X$ is concentrated on a countable set, then $X$ is strongly null.

Theorem 4.7. Assume CH. Then not every strongly null subset of $2^{\omega}$ has the $m_{0}$-property.

Proof. Assume CH. Let $\left\langle T_{\alpha}: \alpha<\omega_{1}\right\rangle$ be an enumeration of all Miller trees and let $\left\langle f_{\alpha}: \alpha<\omega_{1}\right\rangle$ be an enumeration of $\omega^{\omega}$. Construct an increasing sequence $\left\langle M_{\alpha}: \alpha<\omega_{1}\right\rangle$ of countable transitive models of ZFC ${ }^{*}$ such that $f_{\alpha}, T_{\alpha} \in M_{\alpha}$.

Let $G_{\alpha}$ be an $\mathbb{M}$-generic filter over $M_{\alpha}$ such that $T_{\alpha} \in G_{\alpha}$ and let $x_{\alpha} \in \bigcap\{[T]$ : $\left.T \in G_{\alpha}\right\}$. The set $X=\left\{x_{\alpha}: \alpha<\omega_{1}\right\}$ is the one we are looking for.

First observe that $\phi[X]$ is a strongly null subset of $2^{\omega}$. To check this, we will show that it is concentrated on $\mathbb{Q}=\left\{x \in 2^{\omega}: \forall^{\infty} n x(n)=0\right\}$.

Let $U \supseteq \mathbb{Q}$ be open. Then $F=2^{\omega} \backslash U$ is a compact subset of $\phi\left[\omega^{\omega}\right]$ so $F^{\prime}=\phi^{-1}[F]$ is a dominated subset of $\omega^{\omega}$. Let $\alpha \in \omega_{1}$ be such that $F^{\prime} \leq^{*} f_{\alpha}$. Then (as a Miller real is an unbounded real) $\forall \beta>\alpha x_{\beta} \notin F^{\prime}$. Thus $F^{\prime} \cap X$ is countable, so $F \cap \phi[X]$ is countable.

On the other hand, $X$ was designed to intersect $[T]$, for every Miller tree $T$, so it cannot have the $m_{0}$-property. See 7 for similar arguments.

As a consequence, we also get:

Corollary 4.8. Under $\mathrm{CH}$ not every universally null set has the $m_{0}$-property.

Finally, let us notice that such examples cannot be constructed in ZFC.

Proposition 4.9. It is relatively consistent with ZFC that every universally null set has both the $l_{0}$ - and the $m_{0}$-property.

Proof. It is known (see [5] and [6]) that in the model obtained by adding $\omega_{2}$ random reals by the random algebra to a model of $\mathrm{CH}$, every universally null set has cardinality smaller than continuum. On the other hand, one can easily check that every set of cardinality smaller than continuum has both the $l_{0}$ - and the $m_{0}$-property.

Corollary 4.10. It is relatively consistent with ZFC that every strongly null subset of $2^{\omega}$ has both the $l_{0}$ - and the $m_{0}$-property.

Let us also point out that the last corollary follows easily from the Borel Conjecture as well.

\section{REFERENCES}

[1] T. Bartoszyński and H. Judah, Set Theory: On the Structure of the Real Line, A K Peters, Ltd., 1995. MR 96k:03002

[2] J. Brendle, Strolling through paradise, Fundamenta Mathematicae 148 (1995), 1-25. MR 96h:03085

[3] J. B. Brown, The Ramsey sets and related sigma algebras and ideals, Fundamenta Mathematicae 136 (1990), no. 3, 179-185. MR 92a:04003

[4] M. Kysiak, Strongly meager and very meager sets, in preparation.

[5] A. W. Miller, Mapping a set of reals onto the reals, Journal of Symbolic Logic 48 (1983), no. 3, 575-584. MR 84k:03125

[6] _ Special Subsets of the Real Line, in: Handbook of Set-Theoretic Topology (K. Kunen, E. Vaughan, eds.), pp. 201-233, North-Holland, Amsterdam, 1984. MR 86i:54037

[7] A. W. Miller and D. H. Fremlin, On some properties of Hurewicz, Menger, and Rothberger, Fundamenta Mathematicae 129 (1988), no. 1, 17-33. MR 89g:54061 
[8] A. Nowik and T. Weiss, Some remarks on totally imperfect sets, Proceedings of the American Mathematical Society, to appear.

[9] Strongly meager sets and tree forcing notions, Proceedings of the American Mathematical Society 130 (2002), no. 4, 1183-1187. MR 2002j:03049

Institute of Mathematics of the Polish Academy of Sciences, ul. Śniadeckich 8, 00-950 Warszawa, Poland

E-mail address: mkysiak@impan.gov.pl

Institute of Mathematics, WSRP, 08-110 Siedlce, Poland

E-mail address: weiss@wsrp.siedlce.pl 